\title{
Local electrochemical impedance spectroscopy: a window into heterogeneous interfaces
}

\author{
Oumaïma Gharbi, Kieu Ngo, Mireille Turmine and Vincent Vivier*
}

Sorbonne Université, CNRS, Laboratoire Interfaces et Systèmes Electrochimiques, LISE, F-75005 Paris, France

${ }^{*}$ Corresponding author: vincent.vivier@sorbonne-universite.fr

\begin{abstract}
The ability to probe surface reactivity on a local scale has led to new insight on the comprehension of the electrochemical reactivity in relation with the microstructure of the surface. Among the different techniques developed in recent years, local electrochemical impedance spectroscopy has the advantage of using a transient approach to locally characterize a stationary electrochemical system without the need to add any redox mediator in solution, which is a great advantage for the study of different systems.

In this review, particular attention is paid to the different ways of measuring the local impedance, and the technique implementing a local current measurement in solution is deeply discussed. This local electrochemical impedance spectroscopy journey also encompasses a discussion about technical and experimental limitations.
\end{abstract}




\section{Introduction}

The study of the electrochemical interface by means of local measurements is a subject that remains in constant evolution, and is accelerated by the numerous technical developments involving probe size reduction (used to sense the interface reactivity) or the development of more advanced associated electronics for data acquisition. In fact, the versatility offered by local electrochemical techniques benefits a large panel of research topics as different as the local catalytic or photocatalytic activity of materials [1-3], the production of redox species in living cells $[4,5]$, corrosion processes on various interfaces [6-9] or complex kinetics mechanism [10$12]$.

In fact, the term local electrochemical techniques encompasses well established methodologies such as the scanning electrochemical microscopy (SECM) [13, 14] and related techniques [2, 15-18] or scanning vibrating probe technique (SVET) $[19,20]$ which have been widely reviewed these last decades. Among these different techniques, the local electrochemical impedance spectroscopy (LEIS) [21-25] is particularly well-adapted to study complex processes occurring at polarized interfaces. In this short review, we present the different ways of using electrochemical impedance spectroscopy for local measurements focusing on their advantages and drawbacks and the possible improvements will be presented and discussed.

\section{From global to local impedance measurements}

The first approach consisted in developing, on the basis of a global measurement carried out on a sample, the local measurement. Historically, the local current density measurement in solution has been reported in 1960's for corrosion application [26]. It was based on the field strength measurement, in solution, using a twin electrode (i.e. electrode consisting in two reference electrodes immersed in Luggin capillaries). It is worth noting that such measurements could be performed on an externally polarized sample, as well as samples at the open circuit potential for corrosion analysis whereby both anodic and cathodic reactions occur simultaneously. Later in 1970's, it was shown that the use of the SVET [27-29] which is based on the use of a single probe that vibrates in two directions (normal and parallel to the electrode surface) at two different frequencies, improves the quality of the measurement. The key of this technique, which was fully described in a comprehensive paper by Jaffe and Nuccitelli [19], is to perform the measurement of the local potential difference using synchronous detection on the probe's vibration frequencies. These developments have paved the way for local electrochemical impedance spectroscopy (LEIS) by measuring the local current above a polarized sample.

The principle of LEIS was defined in the pioneering work of Isaacs et al. [21,30]. The local current density, $j_{l o c}$, is obtained from the local potential difference in solution (Fig. 1a), $\Delta E_{l o c}$, measured with a probe which consisted in reference microelectrodes positioned close to the interface of interest, as depicted in Fig. 1b. 


$$
j_{l o c}=\frac{\Delta E_{l o c} \cdot \kappa}{d}=\frac{\kappa}{d}\left(\sqrt{\Delta E_{n}^{2}+\Delta E_{r}^{2}}\right)
$$

where $\kappa$ is the electrolyte conductivity, $d$ the distance between the two microelectrodes, $\Delta E_{n}$ and $\Delta E_{r}$ the normal and radial potential differences, respectively. Although it is common to measure the different contributions of the local current density for SVET experiments, it is important to mention that commercial equipments neglected the radial contribution to the current for LEIS measurements. This was due to the poor resolution caused by the use millimetric sized electrodes to probe large samples for which the normal current component is predominant. However, when small samples were studied, such an approximation is no longer valid and the electric field must be measured with a good accuracy using a tri-microelectrode as sketched in Fig. 1b.

The local impedance is then derived from Eq. 1 as

$$
z_{\text {loc }}=\frac{\Delta E_{\text {applied }}(\omega)}{j_{\text {loc }}(\omega)}
$$

where $\Delta E_{\text {applied }}(\omega)$ is the applied voltage perturbation between the substrate (working) and the reference electrodes.

(a)

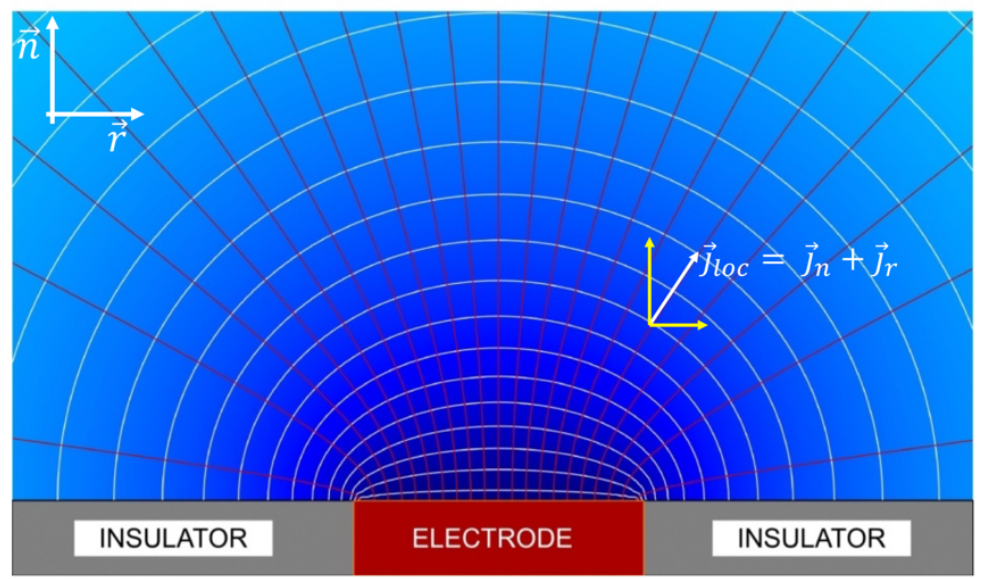

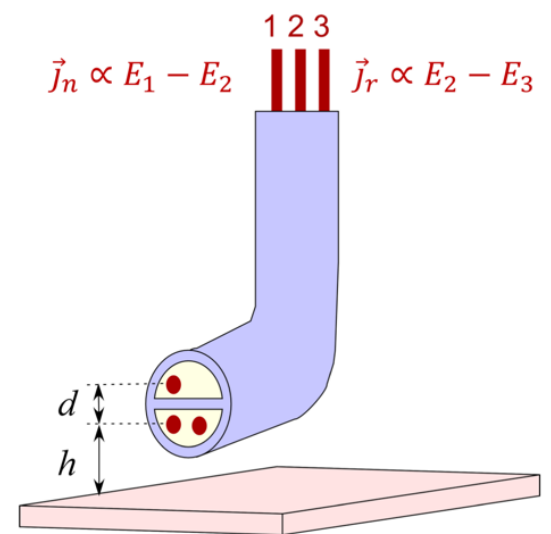

Figure 1: Example of potential (white lines) and current (red lines) distributions in solution close to an electrode embedded in an insulator, and (b) sketch of the probe positioned close to the electrode for measuring the normal $\left(\vec{J}_{n}\right)$ and radial $\left(\vec{J}_{r}\right)$ current contributions from the potential difference between the two vertical and horizontal electrodes, respectively.

Two operating modes are then possible: either perform complete local impedance spectra at different location above the substrate, or perform fixed frequency mapping by moving the probe along a plane parallel to the substrate. Whatever the mode of operation, the spatial resolution of the technique is governed by the following parameters: 
- The size (the diameter) of each microelectrode used for sensing the local potential in solution. This is a general observation common to all techniques using local probe [31].

- The distance between the two sensing microelectrodes. The smaller the distance, the smaller the potential difference. For instance, assuming an impedance of $1 \mathrm{M} \Omega \mathrm{cm}^{2}$ at low frequency limit for the local impedance measured from a sinewave signal of $10 \mathrm{mV}$ amplitude, the local current density to be measured is $10 \mathrm{nA} \mathrm{cm}-2$ (Eq. 2). Assuming that the punctual microreference electrodes are separated by $100 \mu \mathrm{m}$ distance, with an electrolyte conductivity of $50 \mathrm{mS} \mathrm{cm}^{-1}$ (corresponding to a $0.5 \mathrm{M} \mathrm{NaCl}$ solution), the potential to be measured between the two electrodes, given by Eq. 1, is $2 \mathrm{nV}$, only. This is why most of the work reported in the literature is reported on experiments performed with large probe size (i.e. large inter electrode distance) in diluted electrolyte (i.e. low conductivity), thus increasing the potential difference between the two microelectrodes.

- The distance between the probe and the substrate is an important parameter to be considered as well. Indeed, it is necessary to place the probe close to the substrate without having the possibility to regulate its position as a function of the substrate topography, for instance during the mapping of the interface. In addition, the difference in potential to be measured will be smaller if the probe is positioned far from the substrate due to the potential gradient in the electrochemical cell.

Also of note is work by Bayet et al. [25, 32] who reported the use of a SVET for performing LEIS measurements and introduced the notion of simultaneous measurement of local current and local potential (as opposed to using substrate potential) to calculate the local impedance. This approach has been extended by Huang et al. [33-35] who introduced the concept of local interfacial impedance and local ohmic impedance. The latter is particularly interesting as it considers the geometry of the electrode for an improved analysis and interpretation of both local and global impedance diagrams in the high frequency domain [36].

An innovative use of the LEIS is the ability to measure the impedance of a propagating pit by positioning a probe at its mouth, as shown in Fig. 2 in a case of pits formed on $316 \mathrm{~L}$ stainless steel in chloride-containing acidic solution. Interestingly, the figures illustrate two different EIS diagrams that can be easily interpreted using the simplest model of iron dissolution in acidic solution. The curve shape and in particular the low frequency limit of the impedance diagrams clearly reveals that the potential at the bottom of the pit corresponds to a potential on the rise of the activity peak (Fig. 2a) for the lowest chloride content, and on the fall of the activity peak for the highest chloride content (Fig. 2b) as can be deduced from the low frequency limit of the LEIS diagram which tends towards a negative polarization resistance. 

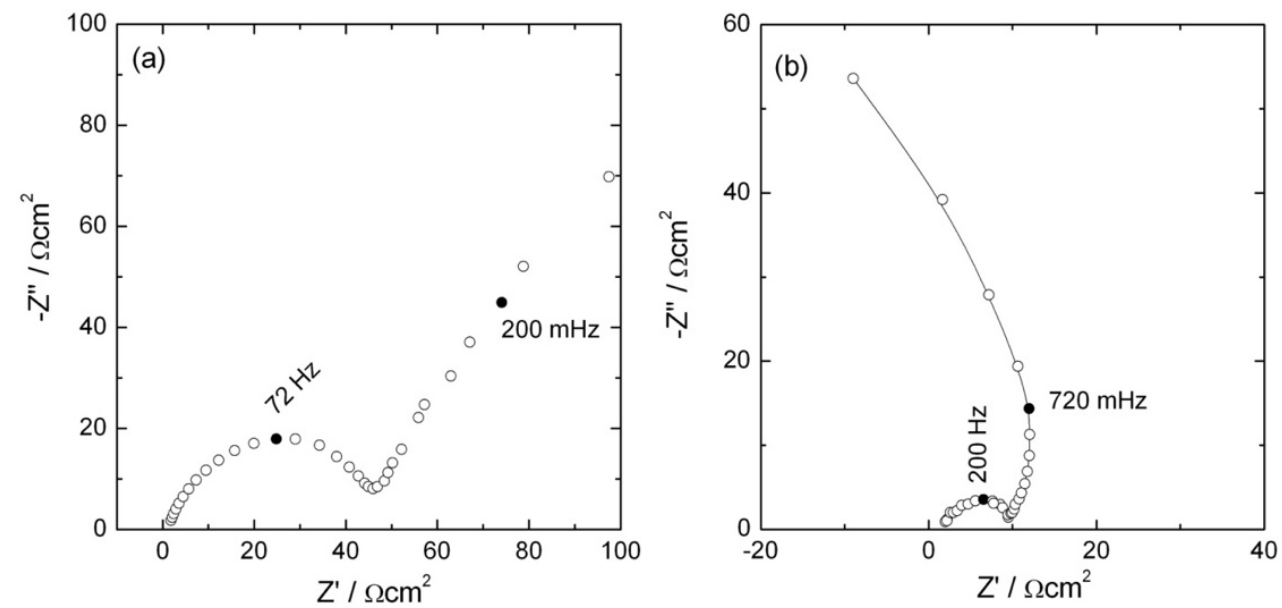

Figure 2: LEIS diagrams obtained above a propagating pit on 316L steel at $0 \mathrm{~V} / \mathrm{SSE}$ in $0.5 \mathrm{M} \mathrm{H}_{2} \mathrm{SO}_{4}$ for (a) $[\mathrm{Cl}]=1 \mathrm{M}$ and (b) $[\mathrm{Cl}]=2 \mathrm{M}$. The two microreference electrodes consisted in $\mathrm{Ag} / \mathrm{AgCl}$ electrode of $100 \mu \mathrm{m}$ in diameter each, separated by $80 \mu \mathrm{m}$.

The LEIS technique is widespread analysis technique used for mapping coatings delamination [37] or disbondment [38] as well as the study of galvanic coupling using the latest developments in dynamic electrochemical impedance spectroscopy [39]. When performing fixed frequency mapping, it is possible to monitor the evolution of defect size over time, but a quantitative analysis at each position requires a complete impedance spectrum to be recorded at each point. This is generally difficult to achieve because the duration of an impedance measurement is governed by the lowest frequencies chosen and the number of points per decade of frequency that is required for the spectrum. Thus, if each measurement lasts at least 10 minutes and a $10 \times 10$ grid is desired, the total duration of the experiment will be 1000 minutes, which is more than 16 hours therefore unrealistic, especially for even slightly dynamic systems.

Today, the best spatial resolution of the LEIS technique is about $10 \mu \mathrm{m}$. However, it should be possible to improve this resolution by decreasing the probe size and the distance between the two microelectrodes and by using an electronic device to cancel the DC offset between them. The latter will allow a higher amplification gain to be used and thus working in concentrated solution with small electrode size. In addition to the probe size, the low-frequency limit of the technique is also governed by the stationarity of the system, similarly to usual EIS measurement. It is also of interest to measure simultaneously global and local impedance responses for an easiest interpretation of the local impedance data. However, interpretation of LEIS and EIS results relies on the same basis and a physico-chemical description of the interface should be preferred.

\section{Alternatives to the LEIS based on impedance spectroscopy}

Local electrochemical techniques in alternating current or potential modes have also experienced a boom with the development of the SECM technique. For instance, applying a 
sine-wave perturbation in the high frequency domain at the tip [40] enables to perform approach curves for positioning the microelectrode close to a substrate whether it is a potentiometric [41] or amperometric $[42,43]$ probe. This technique, which actually consists in measuring the resistance of the electrolyte seen by the probe $[42,44]$, can also be used to perform imaging of a large variety of samples. For instance, the imaging of pitting corrosion [45] or the identification of conductive spots surrounded by an insulated domain [46], or the evolution of surface active area in the case of magnesium corrosion [47], as well as the monitoring of copper dissolution/redeposition between two microelectrodes can be investigated with this technique [48]. In addition, it was also used to control the distance between the tip and the substrate for model neuron and biological substrate imaging $[49,50]$. In that case, the topography of the substrate was obtained from the electrolyte resistance variations. It should also be mentioned that the spatial resolution of this technique is governed by the microelectrode size, thus allowing a better resolution than the LEIS. Interestingly, this technique can be used with high frequency sine-wave perturbation thus allowing fast mapping of the sample.

The impedance measurement of the probe was subsequently extended to a larger frequency domain [51-53]. Indeed, assuming that when the tip-to-substrate distance is short, the impedance of the tip is sensitive to the local surface reactivity of the sample as shown on Fig. 3. As the distance is typically in the range of the electrode diameter, the current and potential distributions are sensitive to the underlying substrate. As a result, the impedance of the tip which is the contribution of the faradaic impedance $\left(Z_{t i p}(\omega)\right)$, and the double layer capacitance, $\left(C_{t i p}\right)$ - depends on the local impedance of the substrate, namely $Z_{s u b}(\omega)$ and $C_{s u b}$ [54]. It was successfully used to characterize oxide thin films [47, 55], localized corrosion [53] as well as selfassembled-monolayer coated electrodes [55], or to distinguish single and double strand DNA [56]. However, although this type of measurement is sensitive to minute changes of the local impedance of the substrate, it is still difficult to perform quantitative analysis of the experimental data as the response depends on both the tip/sample local intrinsic capacitance and the frequency range used [55]. 


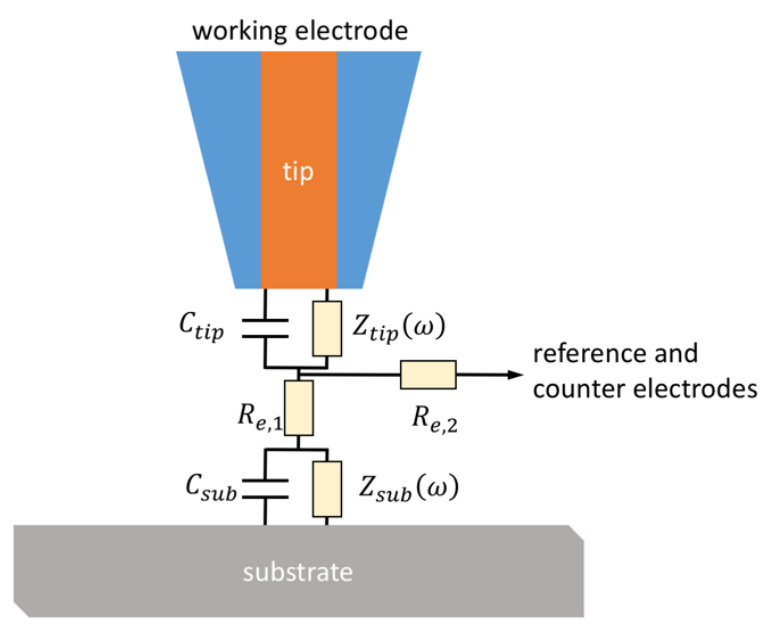

Figure 3: Sketch of the SECM used for performing LEIS measurements. The measurement of the probe impedance is sensitive to the local impedance of the substrate.

A different way of performing local impedance spectroscopy using the SECM was devised for the characterization of the hydrogen evolution reaction at a Pt electrode $[57,58]$. In this application, Trinh et al. used the substrate generator / tip collector mode of the SECM by applying a frequency-depend sinewave perturbation at the substrate to define a complex collection efficiency as the ratio of the current collected at the tip, $\left(i_{\text {tip }}(\omega)\right)$, to the current generated at the substrate, $\left(i_{\text {sub }}(\omega)\right)$. Interestingly, this technique allowed separating the mass transport contribution to the relaxation of adsorbed intermediates involved in the reaction mechanism at the $\mathrm{Pt}$ electrode and allowed the experimental determination of the amount of charge stored at the interface [58]. This ac-SECM mode was also used for the study of iron dissolution mechanisms, hence providing a unique way of direct quantification of $\mathrm{Fe}(\mathrm{I})_{\mathrm{ads}}$ formed at the electrode surface [11]. This technique was shown to be complementary to conventional EIS and permitted the distinction between two iron dissolution mechanisms proposed in acidic solution.

\section{Towards a high resolution measurement: AFM-LEIS}

The use of a conductive tip of an atomic force microscope (AFM) has also been reported as a way for performing local impedance spectroscopy on several systems including the characterization of conductive channels formed on Nafion membrane surface [59] or intergranular corrosion process [60]. From an electric point of view, this technique differs from the ac-SECM as the tip is no longer used as a second working electrode in solution, but as the counter electrode facing the working electrode (substrate), whereas the AFM setup allows the positioning of this counter electrode. The use of a small probe as counter electrode is however detrimental for quantitative analysis since it results in significant perturbation of current and potential distributions above the sample as already pointed out by Lillard et al. [21]. Indeed, from the resolution of Laplace equation for current and potential distributions, the surface area 
sensed with this technique appears to be frequency-dependent as shown in Fig. 4. The simulations performed at $160 \mathrm{kHz}$ and $1.6 \mathrm{~Hz}$ illustrate how the size of the counter electrode and the distance between the counter electrode and the substrate modify the current distribution (i.e. the reactivity) at the substrate surface. These simulations were performed for two frequencies: a high frequency for which the usual faradic impedance at the substrate is expected to be frozen (blocking electrode behavior corresponding to a primary current distribution), and a lowest frequency for which the impedance response is expected to be dominated by the faradaic contribution at the substrate. These simulations show that the current density can vary over orders of magnitude along the substrate radius when comparison is done with the ideal case of an impedance measurement corresponding to a large counter electrode positioned at an infinite distance from the substrate.

(a) $160 \mathrm{kHz}$

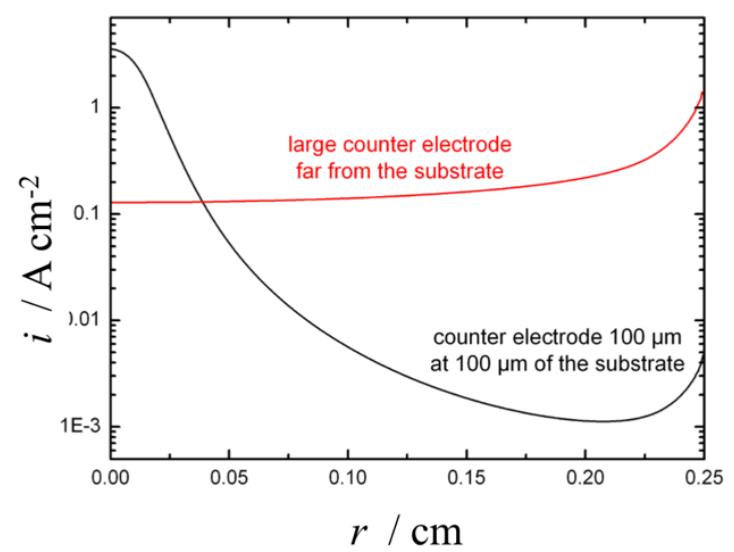

(b) $1.6 \mathrm{~Hz}$

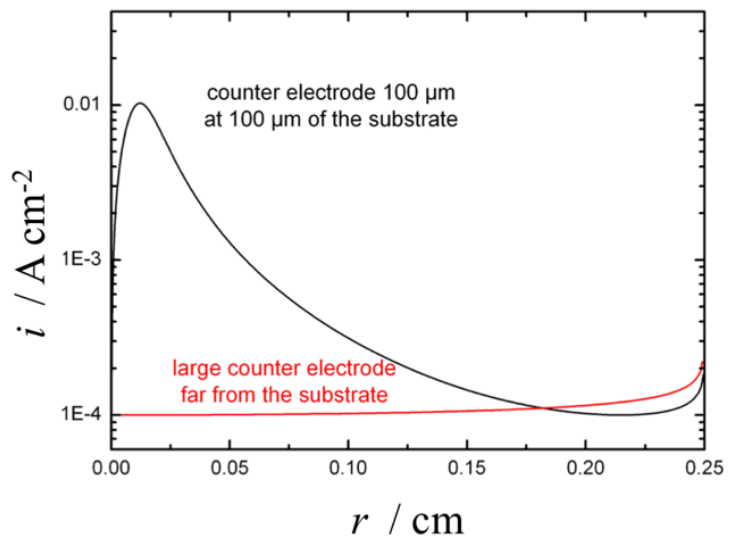

Figure 4: current distribution at a dick electrode for an impedance measurement with a small counter electrode positioned close to the substrate (black curves) and a large counter electrode positioned far from the substrate (red curves) at $160 \mathrm{kHz}$ (a) and $1.6 \mathrm{~Hz}(b)$.

As a result, this technique provides a response in a wide frequency domain that also allows to individualize the different contributions of the electrochemical response, although from a quantitative point of view, the determination of reaction kinetics remains not possible.

\section{Conclusions}

Based on the well-established idea of performing local electrochemical measurements, LEIS provides a powerful tool to probe and track complex reaction mechanisms.

Interestingly, LEIS lies on an impedance measurement performed at the electrode simultaneously with the measurement of local potential differences in solution with two or three microreference electrodes. As a result, conversely to SECM experiments either for positive feedback mode, or for generation / collection mode, there is no need for a redox mediator in solution and LEIS provides a unique way for performing quantitative analysis of the kinetics 
at the substrate. It is also surprising that to date, most of the literature dealing with LEIS is dedicated to corrosion and corrosion protection. Some techniques using the SECM or AFM have been successfully used for evidencing small changes in surface reactivity of the substrate. However, due to the geometry of the system, the analysis of the obtained results remains only qualitative.

The spatial resolution achievable to date is in the order of a few micrometers. This is clearly a possible point of improvement for this technique. In particular, the fabrication of tiny probes is now well-documented in the literature, but the measurement of small potential variations in a large frequency domain requires some new developments in the electronics chain and signal processing. 


\section{References and recommended reading}

Papers of particular interest, published with the period of review, have been highlighted as:

- Paper of special interest.

- Paper of outstanding interest.

[1] A. Botz, J. Clausmeyer, D. Ohl, T. Tarnev, D. Franzen, T. Turek, W. Schuhmann, Local Activities of Hydroxide and Water Determine the Operation of Silver-Based Oxygen

Depolarized Cathodes, Angew Chem Int Ed Engl, 57 (2018) 12285-12289.

[2] F. Conzuelo, K. Sliozberg, R. Gutkowski, S. Grutzke, M. Nebel, W. Schuhmann, High-

Resolution Analysis of Photoanodes for Water Splitting by Means of Scanning

Photoelectrochemical Microscopy, Anal. Chem., 89 (2017) 1222-1228.

[3] J.Y. Kim, H.S. Ahn, A.J. Bard, Surface Interrogation Scanning Electrochemical

Microscopy for a Photoelectrochemical Reaction: Water Oxidation on a Hematite Surface, Anal. Chem., 90 (2018) 3045-3049.

[4] K. Hu, Y. Li, S.A. Rotenberg, C. Amatore, M.V. Mirkin, Electrochemical Measurements of Reactive Oxygen and Nitrogen Species inside Single Phagolysosomes of Living

Macrophages, J. Am. Chem. Soc., 141 (2019) 4564-4568.

[5] Y. Matsumae, Y. Takahashi, H. Shiku, T. Matsue, Quantitative Real-Time Monitoring of Antibody-Induced Internalization of Epidermal Growth Factor Receptor on Single Living Mammalian Cells Using Scanning Electrochemical Microscopy, ChemElectroChem, 5 (2018) 3096-3101.

[6] A. Nazarov, V. Vivier, F. Vucko, D. Thierry, Effect of Tensile Stress on the Passivity Breakdown and Repassivation of AISI 304 Stainless Steel: A Scanning Kelvin Probe and Scanning Electrochemical Microscopy Study, J. Electrochem. Soc., 166 (2019) C3207-C3219. [7] S.S. Jamali, S.E. Moulton, D.E. Tallman, Y. Zhao, J. Weber, G.G. Wallace, Self-healing characteristic of praseodymium conversion coating on AZNd Mg alloy studied by scanning electrochemical microscopy, Electrochem. Commun., 76 (2017) 6-9.

[8] E.L. Silva, S.V. Lamaka, D. Mei, M.L. Zheludkevich, The Reduction of Dissolved Oxygen During Magnesium Corrosion, ChemistryOpen, 7 (2018) 664-668.

[9] D. Snihirova, S.V. Lamaka, Y. Gonzalez-Garcia, A. Yilmaz, N. Scharnagl, M.F.

Montemor, M.L. Zheludkevich, Influence of inhibitor adsorption on readings of microelectrode during SVET measurements, Electrochim. Acta, 322 (2019) 134761.

[10] T. Kai, M. Zhou, S. Johnson, H.S. Ahn, A.J. Bard, Direct Observation of C2O4(*-) and CO2 $\left(_{-}^{*}\right)$ by Oxidation of Oxalate within Nanogap of Scanning Electrochemical Microscope, J. Am. Chem. Soc., 140 (2018) 16178-16183.

[11] D. Trinh, M. Keddam, X.R. Nóvoa, V. Vivier, Characterization of adsorbates by transient measurements in Scanning Electrochemical Microscopy, Electrochim. Acta, 131 (2014) 28-35.

[12] M. Haensch, L. Balboa, M. Graf, A.R. Silva Olaya, J. Weissmüller, G. Wittstock, Mass Transport in Porous Electrodes Studied by Scanning Electrochemical Microscopy: Example of Nanoporous Gold, ChemElectroChem, 6 (2019) 3160-3166.

[13] D. Polcari, P. Dauphin-Ducharme, J. Mauzeroll, Scanning Electrochemical Microscopy: A Comprehensive Review of Experimental Parameters from 1989 to 2015, Chemical Reviews, 116 (2016) 13234-13278. 
[14] T. Kai, C.G. Zoski, A.J. Bard, Scanning electrochemical microscopy at the nanometer level, Chem Commun (Camb), 54 (2018) 1934-1947.

[15] C.H. Chen, K.E. Meadows, A. Cuharuc, S.C. Lai, P.R. Unwin, High resolution mapping of oxygen reduction reaction kinetics at polycrystalline platinum electrodes, Phys. Chem.

Chem. Phys., 16 (2014) 18545-18552.

[16] M.E. Snowden, A.G. Guell, S.C.S. Lai, K. McKelvey, N. Ebejer, M.A. O'Connell, A.W.

Colburn, P.R. Unwin, Scanning Electrochemical Cell Microscopy: Theory and Experiment for Quantitative High Resolution Spatially-Resolved Voltammetry and Simultaneous IonConductance Measurements, Anal. Chem., 84 (2012) 2483-2491.

[17] B. Chen, D. Perry, A. Page, M. Kang, P.R. Unwin, Scanning Ion Conductance Microscopy: Quantitative Nanopipette Delivery-Substrate Electrode Collection Measurements and Mapping, Anal. Chem., 91 (2019) 2516-2524.

[18] E. Daviddi, K.L. Gonos, A.W. Colburn, C.L. Bentley, P.R. Unwin, Scanning Electrochemical Cell Microscopy (SECCM) Chronopotentiometry: Development and Applications in Electroanalysis and Electrocatalysis, Anal. Chem., 91 (2019) 9229-9237.

[19] L.F. Jaffe, R. Nuccitelli, An ultrasensitive vibrating probe for measuring steady extracellular currents, J Cell Biol, 63 (1974) 614-628.

[20] A.C. Bastos, M.C. Quevedo, O.V. Karavai, M.G.S. Ferreira, Review-On the Application of the Scanning Vibrating Electrode Technique (SVET) to Corrosion Research, J. Electrochem. Soc., 164 (2017) C973-C990.

In this work, the authors report explanations about the measurement of local potential in solution to obtain local current density. Authors also provide a detailled description of the experimental procedure for SVET.

[21] R.S. Lillard, P.J. Moran, H.S. Isaacs, A novel method for generating quantitative local electrochemical impedance spectroscopy, J. Electrochem. Soc., 139 (1992) 1007-1012.

The first paper dealing with the LEIS. The concept of local current measurement with a bielectrode instead of using a small counter electrode is introduced for the first time. The experimental results showed the LEIS response for a Mo/Al model system. Authors also introduced LEIS mapping at different frequencies.

[22] V.M.-W. Huang, S.-L. Wu, M.E. Orazem, N. Pebere, B. Tribollet, V. Vivier, Local electrochemical impedance spectroscopy: A review and some recent developments,

Electrochim. Acta, 56 (2011) 8048-8057.

[23] J.B. Jorcin, H. Krawiec, N. Pebere, V. Vignal, Comparison of local electrochemical impedance measurements derived from bi-electrode and microcapillary techniques,

Electrochim. Acta, 54 (2009) 5775-5781.

[24] G. Galicia, N. Pebere, B. Tribollet, V. Vivier, Local and global electrochemical impedances applied to the corrosion behaviour of an AZ91 magnesium alloy, Corros. Sci., 51 (2009) 1789-1794.

[25] E. Bayet, F. Huet, M. Keddam, K. Ogle, H. Takenouti, Local electrochemical impedance measurement: scanning vibrating electrode technique in ac mode, Electrochim. Acta, 44

(1999) 4117-4127. 
[26] I.L. Rosenfeld, I.S. Danilov, Electrochemical aspects of pitting corrosion, Corros. Sci., 7 (1967) 129-142.

[27] H.S. Isaacs, The effect of height on the current distribution measured with a vibrating electrode probe, J. Electrochem. Soc., 138 (1991) 722-728.

[28] M.J. Franklin, D.C. White, H.S. Isaacs, Pitting corrosion by bacteria on carbon steel, determined by the scanning vibrating electrode technique, Corros. Sci., 32 (1991) 945-952. [29] S.V. Lamaka, M. Taryba, M.F. Montemor, H.S. Isaacs, M.G.S. Ferreira, Quasisimultaneous measurements of ionic currents by vibrating probe and $\mathrm{pH}$ distribution by ionselective microelectrode, Electrochem. Commun., 13 (2011) 20-23.

[30] F. Zou, D. Thierry, H.S. Isaacs, A high-resolution probe for localized electrochemical impedance spectroscopy measurements, J. Electrochem. Soc., 144 (1997) 1957-1965.

[31] C.G. Zoski, Review-Advances in Scanning Electrochemical Microscopy (SECM), J. Electrochem. Soc., 163 (2016) H3088-H3100.

[32] E. Bayet, F. Huet, M. Keddam, K. Ogle, H. Takenouti, A novel way of measuring local electrochemical impedance using a single vibrating probe, J. Electrochem. Soc., 144 (1997) L87-L90.

[33] V.M.-W. Huang, V. Vivier, M.E. Orazem, N. Pebere, B. Tribollet, The apparent constant-phase-element behavior of an ideally polarized blocking electrode a global and local impedance analysis, J. Electrochem. Soc., 154 (2007) C81-C88.

[34] V.M.-W. Huang, V. Vivier, I. Frateur, M.E. Orazem, B. Tribollet, The global and local impedance response of a blocking disk electrode with local constant-phase-element behavior, J. Electrochem. Soc., 154 (2007) C89-C98.

[35] V.M.-W. Huang, V. Vivier, M.E. Orazem, N. Pebere, B. Tribollet, The apparent constant-phase-element behavior of a disk electrode with Faradaic reactions. A global and local impedance analysis, J. Electrochem. Soc., 154 (2007) C99-C107.

[36] O. Gharbi, A. Dizon, M.E. Orazem, M.T.T. Tran, B. Tribollet, V. Vivier, From frequency dispersion to ohmic impedance: A new insight on the high-frequency impedance analysis of electrochemical systems, Electrochim. Acta, 320 (2019) 134609.

Based on the LEIS analysis, the authors provide a way to analyse the high frequency of impedance diagrams by taking into account the frequency dispersion.

[37] V. Shkirskiy, P. Volovitch, V. Vivier, Development of quantitative Local Electrochemical Impedance Mapping: an efficient tool for the evaluation of delamination kinetics,

Electrochim. Acta, 235 (2017) 442-452.

This work is devoted to the analysis of LEIS mapping in order to make the technique quantitative.

[38] F. Mahdavi, M. Forsyth, M.Y.J. Tan, Understanding the effects of applied cathodic protection potential and environmental conditions on the rate of cathodic disbondment of coatings by means of local electrochemical measurements on a multi-electrode array, Progress in Organic Coatings, 103 (2017) 83-92.

[39] L. Burczyk, K. Darowicki, Local electrochemical impedance spectroscopy in dynamic mode of galvanic coupling, Electrochim. Acta, 282 (2018) 304-310. 
This work reports on the development of the dynamic LEIS. It shows how to perform simultaneously LEIS and the mapping of the sample in a selected frequency range.

[40] A.S. Baranski, A. Szulborska, High frequency impedance measurements at ultramicroelectrodes, Electrochim. Acta, 41 (1996) 985-991.

[41] B.R. Horrocks, D. Schmidtke, A. Heller, A.J. Bard, Scanning electrochemical microscopy. 24. Enzyme ultramicroelectrodes for the measurement of hydrogen peroxide at surfaces, Anal. Chem., 65 (1993) 3605-3614.

[42] M.A. Alpuche-Aviles, D.O. Wipf, Impedance feedback control for scanning electrochemical microscopy, Anal. Chem., 73 (2001) 4873-4881.

[43] P.M. Diakowski, A.S. Baranski, Positive and negative AC impedance feedback observed above conductive substrates under SECM conditions, Electrochim. Acta, 52 (2006) 854-862.

[44] C. Gabrielli, F. Huet, M. Keddam, P. Rousseau, V. Vivier, Scanning Electrochemical Microscopy Imaging by Means of High-Frequency Impedance Measurements in Feedback Mode, J. Phys. Chem. B, 108 (2004) 11620-11626.

[45] K. Eckhard, M. Etienne, A. Schulte, W. Schuhmann, Constant-distance mode AC-SECM for the visualisation of corrosion pits, Electrochem. Commun., 9 (2007) 1793-1797.

[46] K. Eckhard, H. Shin, B. Mizaikoff, W. Schuhmann, C. Kranz, Alternating current (AC) impedance imaging with combined atomic force scanning electrochemical microscopy (AFMSECM), Electrochem. Commun., 9 (2007) 1311-1315.

[47] G. Baril, G. Galicia, C. Deslouis, N. Pebere, B. Tribollet, V. Vivier, An impedance investigation of the mechanism of pure magnesium corrosion in sodium sulfate solutions, J. Electrochem. Soc., 154 (2007) C108-C113.

[48] C. Gabrielli, E. Ostermann, H. Perrot, V. Vivier, L. Beitone, C. Mace, Concentration mapping around copper microelectrodes studied by scanning electrochemical microscopy, Electrochem. Commun., 7 (2005) 962-968.

[49] J.M. Liebetrau, H.M. Miller, J.E. Baur, S.A. Takacs, V. Anupunpisit, P.A. Garris, D.O. Wipf, Scanning Electrochemical Microscopy of Model Neurons: Imaging and Real-Time Detection of Morphological Changes, Anal. Chem., 75 (2003) 563-571.

[50] J.A. Koch, M.B. Baur, E.L. Woodall, J.E. Baur, Alternating current scanning electrochemical microscopy with simultaneous fast-scan cyclic voltammetry, Anal. Chem., 84 (2012) 9537-9543.

[51] A.S. Bandarenka, K. Eckhard, A. Maljusch, W. Schuhmann, Localized electrochemical impedance spectroscopy: visualization of spatial distributions of the key parameters describing solid/liquid interfaces, Anal. Chem., 85 (2013) 2443-2448.

[52] A.S. Bandarenka, A. Maljusch, V. Kuznetsov, K. Eckhard, W. Schuhmann, Localized Impedance Measurements for Electrochemical Surface Science, J. Phys. Chem. C, 118 (2014) 8952-8959.

[53] V. Kuznetsov, A. Maljusch, R.M. Souto, A.S. Bandarenka, W. Schuhmann, Characterisation of localised corrosion processes using scanning electrochemical impedance microscopy, Electrochem. Commun., 44 (2014) 38-41.

[54] A.S. Baranski, P.M. Diakowski, Application of AC impedance techniques to Scanning Electrochemical Microscopy, Journal of Solid State Electrochemistry, 8 (2004) 683-692. 
[55] A. Estrada-Vargas, A. Bandarenka, V. Kuznetsov, W. Schuhmann, In Situ Characterization of Ultrathin Films by Scanning Electrochemical Impedance Microscopy, Anal. Chem., 88 (2016) 3354-3362.

Authors report a comprehensive analysis of the scanning electrochemical impedance microscopy for different systems.

[56] A. Estrada-Vargas, D. Jambrec, Y.U. Kayran, V. Kuznetsov, W. Schuhmann, Differentiation between Single- and Double-Stranded DNA through Local Capacitance Measurements, ChemElectroChem, 3 (2016) 855-857.

[57] D. Trinh, M. Keddam, X.R. Novoa, V. Vivier, Alternating-Current Measurements in Scanning Electrochemical Microscopy, Part 1: Principle and Theory, ChemPhysChem, 12 (2011) 2169-2176.

[58] D. Trinh, M. Keddam, X.R. Novoa, V. Vivier, Alternating Current Measurements in Scanning Electrochemical Microscopy, Part 2: Detection of Adsorbates, ChemPhysChem, 12 (2011) 2177-2183.

[59] X. Wang, B.T. Habte, S. Zhang, H. Yang, J. Zhao, F. Jiang, Q. He, Localized Electrochemical Impedance Measurements on Nafion Membranes: Observation and Analysis of Spatially Diverse Proton Transport Using Atomic Force Microscopy, Anal. Chem., 91 (2019) 11678-11686.

[60] A. Arutunow, K. Darowicki, M.T. Tobiszewski, Electrical mapping of AISI 304 stainless steel subjected to intergranular corrosion performed by means of AFM-LIS in the contact mode, Corros. Sci., 71 (2013) 37-42. 